\title{
Cell migration in paediatric glioma; characterisation and potential therapeutic targeting
}

\author{
J V Cockle ${ }^{* 1,2}$, S Picton ${ }^{2}$, J Levesley ${ }^{1}$, E llett ${ }^{1}$, A M Carcaboso ${ }^{3}$, S Short ${ }^{1}$, L P Steel ${ }^{1}$, A Melcher $^{1}$, S E Lawler $^{4}$ \\ and A Brüning-Richardson ${ }^{1}$ \\ ${ }^{1}$ Leeds Institute of Cancer Studies and Pathology, University of Leeds, Wellcome Trust Brenner Building, St James's University \\ Hospital, Leeds, LS9 7TF, UK; ${ }^{2}$ Yorkshire Regional Centre for Paediatric Oncology and Haematology, Leeds General Infirmary, \\ Great George Street, Leeds, LS1 3EX, UK; ${ }^{3}$ Preclinical Therapeutics and Drug Delivery Research Program, Department of \\ Oncology, Hospital Sant Joan de Déu Barcelona, Preclinical Therapeutics and Drug Delivery Research Program Santa Rosa, 39-57, \\ 4th floor 08950 Esplugues de Llobregat, Barcelona, Spain and ${ }^{4}$ Department of Neurosurgery, Brigham and Women's Hospital, \\ Harvard Medical School, 4 Blackfan Circle, HIM 930A, Boston, MA, 02115, USA
}

Background: Paediatric high grade glioma (pHGG) and diffuse intrinsic pontine glioma (DIPG) are highly aggressive brain tumours. Their invasive phenotype contributes to their limited therapeutic response, and novel treatments that block brain tumour invasion are needed.

Methods: Here, we examine the migratory characteristics and treatment effect of small molecule glycogen synthase kinase-3 inhibitors, lithium chloride $(\mathrm{LiCl})$ and the indirubin derivative 6-bromoindirubin-oxime $(\mathrm{BIO})$, previously shown to inhibit the migration of adult glioma cells, on two pHGG cell lines (SF188 and KNS42) and one patient-derived DIPG line (HSJD-DIPG-007) using 2D (transwell membrane, immunofluorescence, live cell imaging) and 3D (migration on nanofibre plates and spheroid invasion in collagen) assays.

Results: All lines were migratory, but there were differences in morphology and migration rates. Both $\mathrm{LiCl}$ and $\mathrm{BIO}$ reduced migration and instigated cytoskeletal rearrangement of stress fibres and focal adhesions when viewed by immunofluorescence. In the presence of drugs, loss of polarity and differences in cellular movement were observed by live cell imaging.

Conclusions: Ours is the first study to demonstrate that it is possible to pharmacologically target migration of paediatric glioma in vitro using $\mathrm{LiCl}$ and $\mathrm{BIO}$, and we conclude that these agents and their derivatives warrant further preclinical investigation as potential anti-migratory therapeutics for these devastating tumours.

Paediatric high grade glioma (pHGG) and diffuse intrinsic pontine glioma (DIPG) are highly aggressive tumours associated with dismal prognosis. Paediatric high grade gliomas account for $8-12 \%$ of all primary paediatric CNS tumours (Jones et al, 2012). Current treatment regimens involve maximal surgical resection, radiation therapy and chemotherapy. However, despite this intensive treatment approach, 5-year survival remains between 15 and 35\% (Broniscer and Gajjar, 2004). Diffuse intrinsic pontine gliomas are the most common brainstem tumours of childhood and represent one of the most challenging paediatric tumours to treat. Surgery is not an option, chemotherapeutics have failed to improve outcomes, and radiotherapy can only temporarily slow disease progression, which is associated with median survival of less than 1 year (Warren, 2012). Both pHGG and DIPG are known to have diffuse infiltrative growth patterns, and this invasive phenotype contributes to their limited therapeutic response (Demuth and

*Correspondence: Dr JV Cockle; E-mail: j.v.cockle@leeds.ac.uk

Revised 12 December 2014; accepted 17 December 2014; published online 27 January 2015

(c) 2015 Cancer Research UK. All rights reserved 0007-0920/15 
Berens, 2004; Louis, 2006). As a result, there is a pressing clinical need to develop novel therapeutic approaches that effectively reduce such paediatric brain tumour migration and invasion.

Adult HGG cells are known to migrate in specific patterns, following the orientation of white matter tracts, capillaries and unmyelinated axons (Louis, 2006; Agudelo-Garcia et al, 2011). Recent studies have suggested that glycogen synthase kinase-3 (GSK-3), a serine/threonine protein kinase, plays a key role in orchestrating cell migration, through regulating cellular structure, motility and dynamics of the cytoskeleton (Grimes and Jope, 2001; Wakefield et al, 2003; Sun et al, 2009). The effects of small molecule GSK-3 inhibitors on blocking adult glioma migration and invasion have been demonstrated both in vitro and in vivo (Nowicki et al, 2008; Williams et al, 2011) and offer a potential novel anti-invasive approach for glioma. The effects of GSK-3 inhibitors on pHGG and DIPG migration and invasion are unknown, and given the distinct biological and clinical phenotype of the adult and paediatric diseases (Jones et al, 2012), warrant separate investigation.

Very few studies have focused on identifying existing or novel therapeutic agents that are capable of impairing migration and invasion in pHGG and DIPG owing to limited patient tissue availability. We have conducted a comprehensive analysis of cell migration in two pHGG cell lines and one rare patient-derived DIPG cell line using a range of $2 \mathrm{D}$ and $3 \mathrm{D}$ assays. In addition, we also demonstrate for the first time that treatment with the small molecule GSK-3 inhibitors, lithium chloride ( $\mathrm{LiCl}$ ) and the indirubin derivative 6-bromoindirubin-oxime (BIO), which have previously been shown to block migration of adult glioma cells (Nowicki et al, 2008; Williams et al, 2011), also inhibit the migration of pHGG and DIPG cells in vitro. These agents represent novel anti-invasive candidates that may improve the clinical management of these challenging diseases in children.

\section{MATERIALS AND METHODS}

Cell lines and reagents. Paediatric glioma cell lines SF188 (Grade IV, Glioblastoma) and KNS42 (Grade IV, Glioblastoma) were obtained from Dr Chris Jones (Institute of Cancer Research, London, UK). Cells were grown in Dulbecco's Modified Eagles' medium (Sigma-Aldrich, Dorset, UK) with 10\% heat-inactivated foetal calf serum (Labtech, East Sussex, UK) and 0.5\% penicillinstreptomycin (Sigma-Aldrich). Cell line identity was verified by serial tandem repeat profiling at the University of Leeds. The patient autopsy-derived DIPG cell line HSJD-DIPG-007 was obtained from Dr Angel M. Carcaboso (Hospital Sant Joan de Déu Barcelona, Barcelona, Spain). Cells were cultured as tumour spheres in tumour stem base medium (Life Technologies, Paisley, UK) supplemented with B-27 (Life technologies) and human growth factors EGF, FGF-basic, PDGF-AA and PDGF-BB (Peprotech, Rocky Hill, NJ, USA). All cell lines were free of mycoplasma contamination.

Reagents used were LiCl (Sigma-Aldrich) and BIO (Calbiochem, Nottingham, UK).

Three-dimensional cell migration/invasion assay. For threedimensional spheroid cultures, $1 \times 10^{3}$ cells were incubated for $72 \mathrm{~h}$ in an ultra-low attachment round bottom 96-well plate (Costar, Corning Lifesciences, Tewksbury, MA, USA). Aggregates were then encased in $100 \mu \mathrm{l}$ of rat tail collagen-1 (BD Biosciences) neutralised with $1 \mathrm{~m}$ sodium hydroxide $(\mathrm{NaOH})$ in $5 \times$ Dulbecco's Modified Eagles' medium. After polymerisation at $37^{\circ} \mathrm{C}$, the collagen was overlaid with $100 \mu \mathrm{l}$ of standard growth medium \pm GSK-3 inhibitors over a range of concentrations. Spheroid expansion and invasion into the collagen matrix was monitored for $72 \mathrm{~h}$ using the EVOS cell imaging system (Advanced Microscopy
Group, Life Technologies) and analysed with Volocity 3D Image Analysis software (Perkin-Elmer Inc., Coventry, UK) and Image J (http://rsbweb.nih.gov/ij). In order to quantify migration, two zones of migration were defined: the invasion zone represents the area outside the spheroid core to where approximately $75 \%$ of migrating cells invaded into, whereas the leading edge zone represents the total area containing migrated cells (Supplementary Figure 1). This method has been previously described (Ma et al, 2011) and improved the accuracy of the analysis. The migration index was calculated as

$$
\frac{\text { area of zone }- \text { area of core }}{\text { total area }}
$$

Transwell migration assay. Transwell assays were performed using $8 \mu \mathrm{m}$ pore inserts (Greiner Bio-One, Stroud, UK) pre-coated with $5 \mu \mathrm{g} \mathrm{ml}^{-1}$ of the chemo-attractant fibronectin (Sigma). Cell lines were pre-treated $\pm 20 \mathrm{~mm} \mathrm{LiCl}$ or $5 \mu \mathrm{M}$ BIO for $1 \mathrm{~h}$. Cells $\left(5 \times 10^{4}\right)$ were then placed into transwell inserts in triplicate and allowed to migrate for $4 \mathrm{~h}$. Migrated cells were fixed with $1 \%$ glutaraldehyde (Sigma-Aldrich) in PBS and visualised by staining with $0.1 \mu \mathrm{g} \mathrm{ml}^{-1}$ DAPI (Biotium, Hayward, CA, USA). Migration was determined by imaging DAPI nuclear stain with the EVOS imaging system and quantifying the fluorescence signal for the individual transwells using Volocity 3D Image Analysis software.

Migration on nanofibre scaffolds. To evaluate migration on $3 \mathrm{D}$ nanofibre scaffolds, $1 \times 10^{3}$ cells labelled with Cell Tracker Green dye (Invitrogen, Paisley, UK) were cultured as hanging drops as described (Del Duca et al, 2004). Spheroid aggregates were transferred into wells of aligned poly- $\varepsilon$-caprolactone nanofibrecoated culture plates (Nanofibre solutions, Columbus, OH, USA), which allows cell migration on a 3D scaffold stimulated by topographical cues (Johnson et al, 2009). Spheroids were incubated at $37^{\circ} \mathrm{C}$ in growth medium $\pm 20 \mathrm{~mm} \mathrm{LiCl}$ or $5 \mu \mathrm{M} \mathrm{BIO}$ in triplicate and migration was monitored for $72 \mathrm{~h}$ using the EVOS imaging system and quantified using Image J software.

Immunofluorescence (IF) and western blotting. Antibodies for IF studies were rat anti-alpha tubulin monoclonal antibody (Serotec, Kidlington, UK), rabbit anti- $\beta$-catenin antibody (Sigma-Aldrich) and secondary antibodies (Alexa Fluor-conjugated reagents obtained from Molecular Probes, Paisley, UK). For the identification of focal adhesions, a staining kit (Millipore, Nottingham, UK) was used. Actin filament and nuclear staining was performed with rhodamine-phalloidin (Molecular Probes, Life Technologies) and DAPI (Molecular Probes, Life Technologies) at recommended working concentrations. For western blotting, proteins $(40 \mu \mathrm{g})$ were separated by SDS-PAGE and transferred to nitrocellulose. Antibodies used were anti-GSK-3 $\beta$, anti-phosphoGSK-3 $\beta$ (Ser9) (Cell Signaling, Danvers, MA, USA), anti-GSK$3 \alpha+\beta$ (phospho Y279+Y216) (Abcam, Cambridge, UK) and secondary antibodies as per manufacturer instructions (Dako, Cambridgeshire, UK).

Live cell imaging. Cells in $500 \mu$ l of growth medium $\pm 20 \mathrm{~mm} \mathrm{LiCl}$ or $5 \mu \mathrm{M}$ BIO were placed in each quarter of an Ibidi imaging dish (Nikon, Düsseldorf, Germany) and cultured in the incubation/ imaging chamber of the Nikon Biostation IM time lapse imaging system. Cells were imaged for $24 \mathrm{~h}$ at 3 -min intervals at $37^{\circ} \mathrm{C}$ with $5 \% \mathrm{CO}_{2}$ in air. The Biostation IM associated software was used to create movie files for analysis. Cell tracking was performed in Image J with MTrackJ (www.imagescience.org/meijering/software/ mtrackj/). For tracking, the nucleus of each cell was identified and tracked over $24 \mathrm{~h}$ at $150 \mathrm{~min}$ intervals.

Cell viability and proliferation analysis. Cell viability of both monolayers and spheroid aggregates incubated with growth medium \pm GSK-3 inhibitors were measured using the WST-1 
assay (Roche, West Sussex, UK) in 96 well plates, according to the manufacturer's recommendations. Tumour spheroids were imaged at $24 \mathrm{~h}, 48 \mathrm{~h}$ and $72 \mathrm{~h}$ using the EVOS cell imaging system and spheroid core area was calculated using Image $\mathrm{J}$ software to evaluate proliferation over time.

Statistical analysis. Statistical analysis was carried out using SPSS version 21 (IBM, New York, NY, USA). Statistical significance between multiple groups was determined by ANOVA. $P$-values $<0.05$ were considered statistically significant.

\section{RESULTS}

Paediatric glioma cell lines form tumour spheroids displaying different patterns of migration. In this study, we sought to identify chemotherapeutic agents capable of impairing migration and invasion in pHGG and DIPG cell lines. Tumour spheroids represent a useful model for examining tumour biology in vitro as they are three dimensional and comprise of a surface with ready access to nutrients and oxygen and an inner hypoxic core (Nowicki et al, 2008). Moreover, once embedded in a matrix such as collagen, cells can migrate outward from the core representing a model of the process of invasion (Nowicki et al, 2008).

Two pHGG cell lines, SF188 (glioblastoma) and KNS42 (glioblastoma) and one patient-derived DIPG cell line, HSJDDIPG-007, were first examined for their ability to form spheroids in culture. Similar to Vinci et al (2012), we noted that all three cell lines readily formed round dense spheroids within $24 \mathrm{~h}$ when cultured in low adherence 96-well round bottomed plates (Figure 1A). Paediatric glioma tumour spheroids were then embedded in collagen, and cell migration was monitored over $72 \mathrm{~h}$ by light microscopy. The cell lines exhibited distinct migratory characteristics and migration patterns were strikingly different (Figure 1B): SF188 displayed a cogwheel pattern of migration with what appeared to be long thin symmetrical protrusions branching from the central core, whereas KNS42 and HSJD-DIPG-007 migrated by extending flattened protrusions and spreading in a sheet-like manner. The observed differences were also reflected in the migration indices obtained for the migration edge for each cell; KNS42 migrated significantly less than SF188 and HSJD-DIPG007 (migration index 0.59). No significant difference was observed between the migration index of SF188 (0.87) and HSJD-DIPG-007 (0.78) (Figure 1C).

$\mathrm{LiCl}$ and $\mathrm{BIO}$ inhibit migration and invasion of paediatric glioma cells in 3D spheroid assays. The small molecule GSK-3 inhibitors, $\mathrm{LiCl}$ and the indirubin derivative $\mathrm{BIO}$, have recently been shown to block migration in adult glioblastoma cell lines (Nowicki et al, 2008; Williams et al, 2011). Moreover, indirubin derivatives extend survival in animal glioblastoma orthotopic xenograft models and block metastasis in vivo (Williams et al, 2011). However, whether these agents exert similar effects upon migration and invasion in pHGG and DIPG cell lines has not been previously determined. In order to answer this question, tumour spheres from each cell line were embedded in collagen, overlaid
A





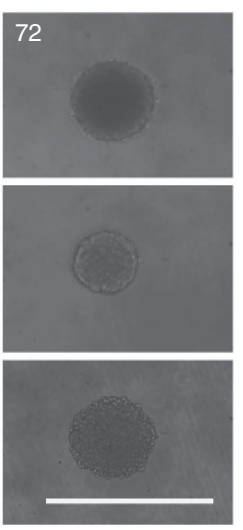

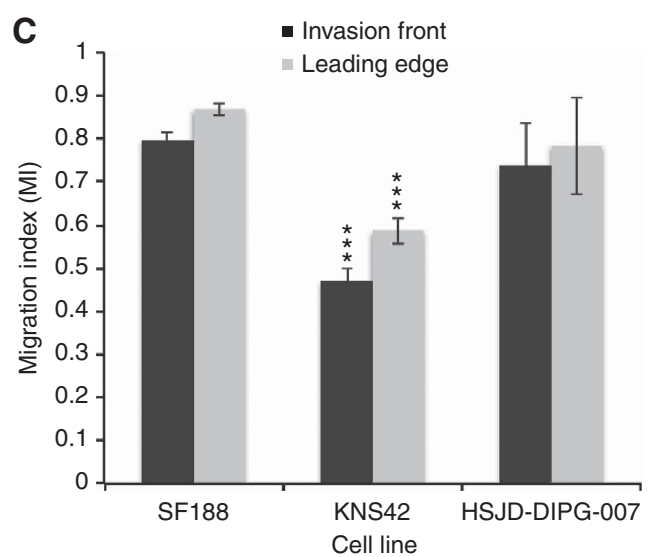

B

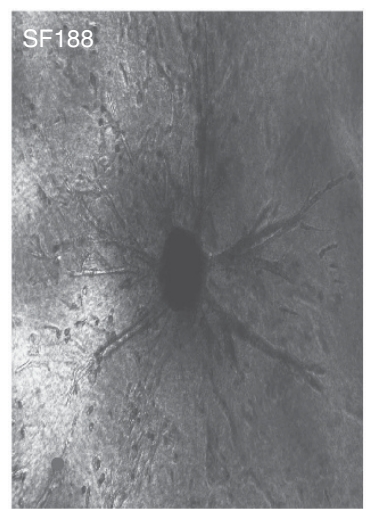

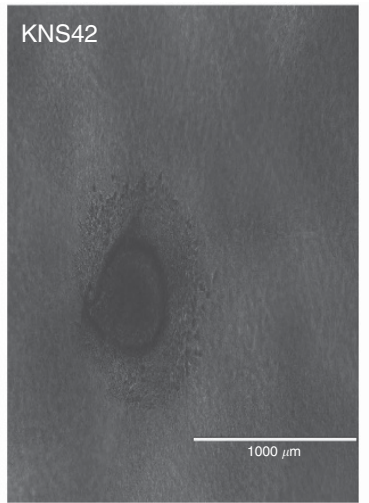



Figure 1. Paediatric glioma cell lines readily form tumour spheres from monolayers and demonstrate different patterns of migration.

(A) The paediatric glioblastoma cell lines SF188 and KNS42 and the patient-derived DIPG cell line HSJD-DIPG-007 were evaluated for their ability to form tumour spheres in low adherent 96 well round-bottomed plates. After $72 \mathrm{~h}$ of incubation, all three cell lines formed tumour spheres. Images at $\times 100$ magnification. (B) SF188, KNS42 and HSJD-DIPG-007 tumour spheroids were capable of migrating after embedding in a collagen matrix as demonstrated at time point $72 \mathrm{~h}$. Images at $\times 40$ magnification, scale bar $=1000 \mu \mathrm{m}$. (C) The paediatric glioma cell lines differed in their ability to migrate as determined by the migration index (MI) for the invasion front and the leading edge. Error bars expressed as \pm s.e.m. ${ }^{\star \star \star} P<0.001$ by $T$ test. Results are representative of $n=3$ individual experiments. 
with the drugs at varying concentrations and migration and invasion were imaged over $72 \mathrm{~h}$ (Figure $2 \mathrm{~A}$ and $\mathrm{B}$ ). A migration index was then calculated for each condition to compare the invasion front and leading edge zone for each treatment (Figure 2C).
We found that $\mathrm{LiCl}$ and $\mathrm{BIO}$ reduced migration and invasion in a concentration-dependent manner (Figure 2C). After $72 \mathrm{~h}, 10$ or $5 \mu \mathrm{M}$ BIO significantly blocked invasion of both pHGG cell lines (Figure 2C) (mean \pm s.e.m. SF188 leading edge; control medium $0.869 \pm 0.014, \quad$ BIO $10 \mu \mathrm{M} \quad 0.292 \pm 0.069 \quad P<0.001, \quad$ BIO $5 \mu \mathrm{M}$

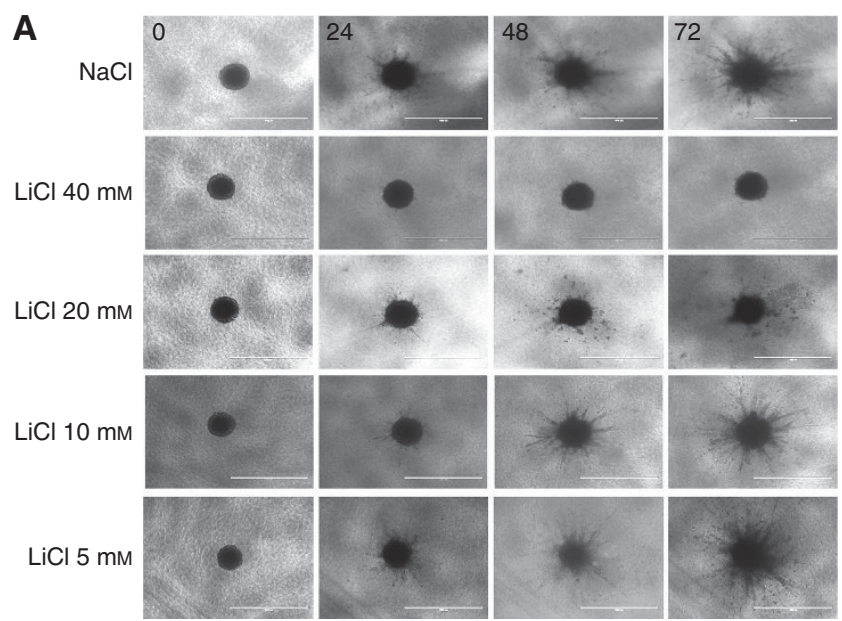

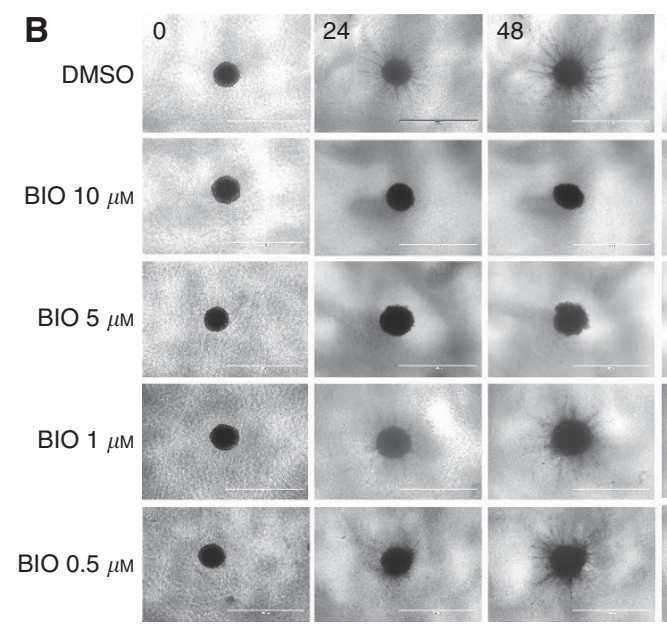

- Invasion zone - Leading edge

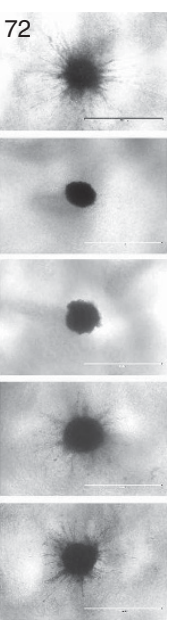

- Invasion zone - Leading edge
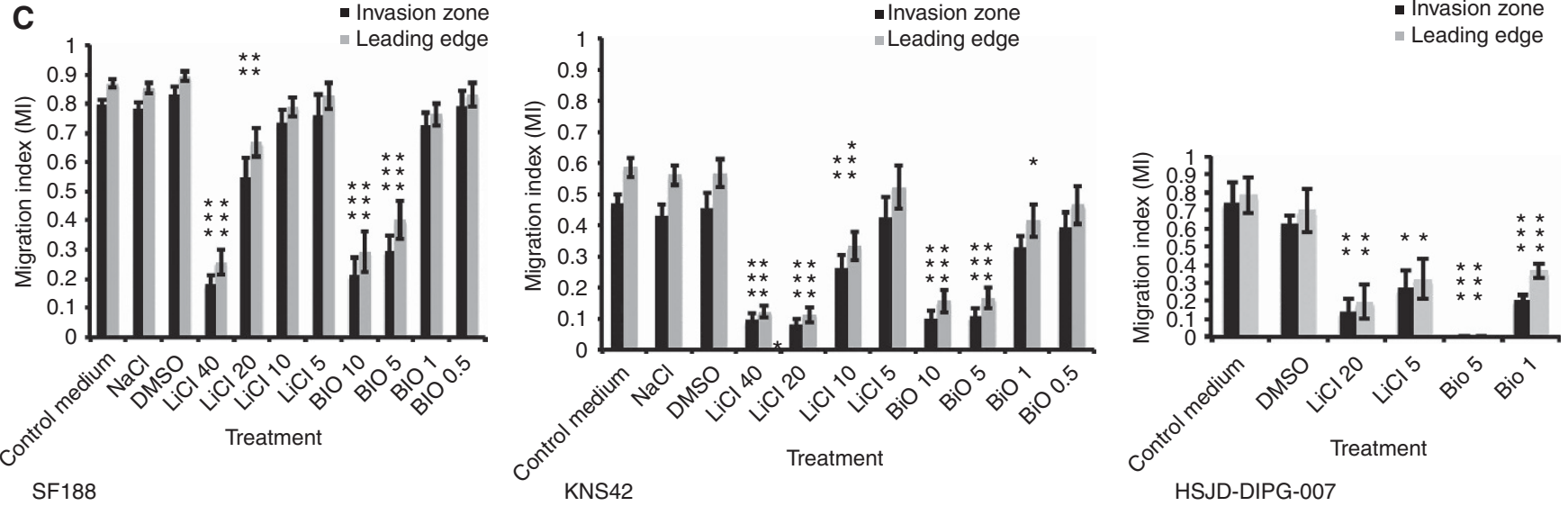

HSJD-DIPG-007


Figure 2. Inhibition of paediatric glioma tumour sphere invasion by $\mathrm{LiCl}$ and $\mathrm{BIO}$. Tumour spheroids (SF188) were embedded in collagen and incubated in (A) $\mathrm{LiCl}$ at 40,20,10,5 mM in $\mathrm{NaCl}$ or (B) BIO at 10,5, 1, $0.5 \mu \mathrm{m}$ in DMSO for $72 \mathrm{~h}$. Migration was inhibited by both $\mathrm{LiCl}$ and $\mathrm{BIO}$ as shown in representative stills at 24,48 and $72 \mathrm{~h}$ using the EVOS cell imaging system at $\times 40$ magnification. Scale bar $=1000 \mu \mathrm{m}$.

(C) Quantitative analysis of migration inhibition confirmed the results visualised by microscopy. Control medium used was Dulbecco's Modified Eagles' medium plus $10 \%$ heat-inactivated foetal calf serum. Both $\mathrm{LiCl}$ and $\mathrm{BIO}$ had statistically significant anti-migratory effects on the invasion zone and leading edge as indicated by * on all three cell lines (SF188, KNS42 and HSJD-DIPG-007). Results are representative of $n=3$ individual experiments. Error bars expressed as \pm s.e.m. ${ }^{\star} P<0.05,{ }^{\star} * P<0.01,{ }^{\star \star \star} P<0.001$ by ANOVA for each cell type and treatment. (D) Cell viability of paediatric glioma lines (SF188 and KNS42) grown as tumour spheroids treated with various concentrations of $\mathrm{LiCl}$ and $\mathrm{BIO}$ was determined by WST-1 assay and then expressed as a percentage of controls. Error bars expressed as \pm s.e.m. The effects of LiCl and $\mathrm{BIO}$ on $\beta$-catenin localisation were evaluated for SF188 (E) and KNS42 (F) compared with medium mock-treated controls by immunofluorescent labelling. Arrows indicate $\beta$-catenin localisation. Red labelling- $\beta$-catenin, green labeling-actin, blue labeling-DAPI staining. Magnification $\times 63$. 

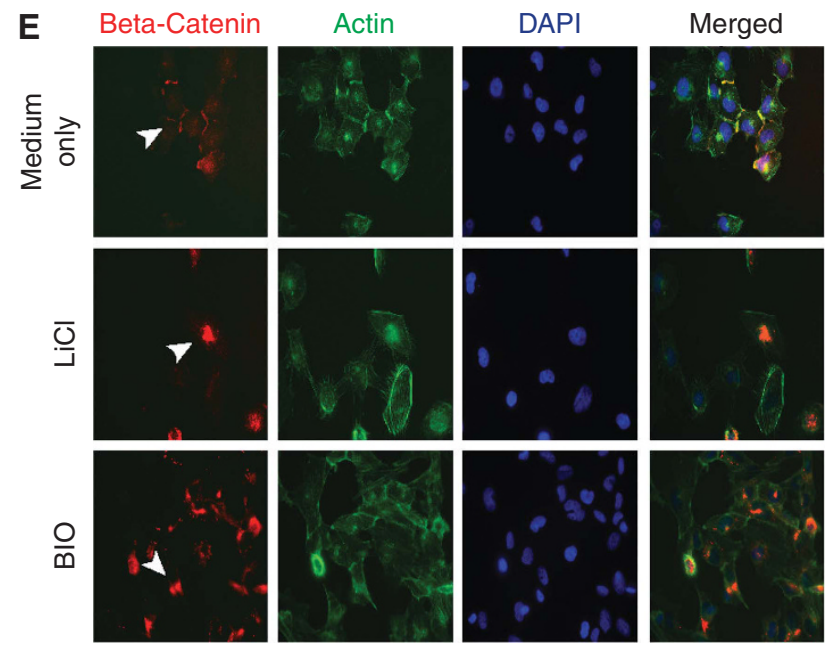

SF188

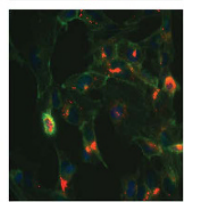

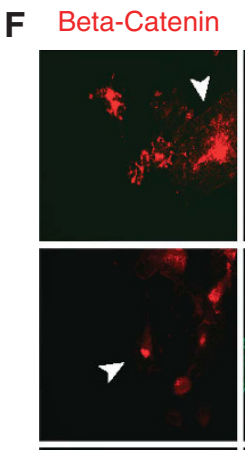


KNS42

Figure 2. (Continued)

$0.401 \pm 0.065 P<0.001$ KNS42 leading edge; control medium $0.587 \pm 0.31, \quad$ BIO $10 \mu \mathrm{M} \quad 0.157 \pm 0.036 \quad P<0.001, \quad$ BIO $5 \mu \mathrm{M}$ $0.166 \pm 0.033 P<0.001)$. The invasion of the DIPG cell line at $72 \mathrm{~h}$ was significantly blocked by $\mathrm{BIO}$ at both 5 and $1 \mu \mathrm{M}$ (mean \pm s.e.m. HSJD-DIPG-007 leading edge; control medium $0.784 \pm 0.1$, BIO $5 \mu \mathrm{M} \quad 0.0 \quad P<0.001$, BIO $1 \mu \mathrm{M} \quad 0.36 \pm 0.040$ $P<0.001)$. Treatment with 40 and $20 \mathrm{~mm} \mathrm{LiCl}$ for $72 \mathrm{~h}$ also caused a statistically significant reduction of invasion for SF188 and KNS42 (for both the invasion front and leading edge (Figure 2C)) (mean \pm s.e.m. SF188 leading edge; control medium $0.869 \pm 0.014$, $\mathrm{LiCl} \quad 40 \mathrm{~mm} \quad 0.256 \pm 0.042 P<0.001, \quad \mathrm{LiCl} 20 \mathrm{~mm} \quad 0.667 \pm 0.05$ $P=0.005$, KNS42 control medium $0.587 \pm 0.31, \mathrm{LiCl} 40 \mathrm{~mm}$ $0.123 \pm 0.02 P<0.001, \mathrm{LiCl} 20 \mathrm{~mm} 0.112 \pm 0.026 P<0.001) . \mathrm{LiCl}$ at $20 \mathrm{~mm}$ and $5 \mathrm{~mm}$ significantly reduced the invasion of HSJDDIPG-007 following $72 \mathrm{~h}$ of treatment (mean \pm s.e.m. HSID-DIPG007 leading edge; control medium $0.784 \pm 0.1, \mathrm{LiCl} 20 \mathrm{~mm}$ $0.19 \pm 0.92 P<0.01$, LiCl 5 mм $0.322 \pm 0.11 P<0.05)$.

To confirm that the observed effects on migration after drug treatment were the result of specific anti-migratory effects and not caused by non-specific cytotoxic or anti-proliferative effects, we investigated the effects of the two inhibitors on spheroid viability by WST-1 assays. This assay has previously been used to determine the viability of cells grown as spheroids (Van Beusechem et al, 2003). In the 3D spheroid system, both pHGG cell lines displayed viability of at least $75 \%$ or above for all treatments with $\mathrm{LiCl}$ and BIO (Figure 2D). Proliferation, as determined by spheroid core size changed over time, was only reduced at $40 \mathrm{~mm} \mathrm{LiCl}$ in SF188 (Supplementary Figure 2).

The effect of $\mathrm{LiCl}$ and $\mathrm{BIO}$ on migration has previously been investigated in adult glioma and their anti-migratory activity has been attributed to their ability to inhibit GSK-3 and stabilise $\beta$-catenin (Nowicki et al, 2008; Williams et al, 2011). In order to confirm that $\mathrm{LiCl}$ and $\mathrm{BIO}$ target GSK-3 in pHGG, we carried out western blot analysis of the activated and inactivated forms of GSK-3 protein. $\mathrm{LiCl}$ increased Ser9 phosphorylated GSK-3 $\beta$ (inactivated form) and $\mathrm{BIO}$ decreased the activating tyrosine of GSK-3 $\alpha \beta$ (Supplementary Figure 3). Next, we examined $\beta$-catenin localisation and staining by IF post treatment (Figure 2E and F). For SF188, cells treated with both $\mathrm{LiCl}$ or $\mathrm{BIO}$ displayed reduced staining of $\beta$-catenin at the plasma membrane with enhanced cytoplasmic and perinuclear staining. This was more marked for cells treated with BIO than LiCl (Figure 2E). For KNS42, surface and intracellular staining of $\beta$-catenin was observed in the control; however, $\mathrm{BIO}$ and $\mathrm{LiCl}$ treatment resulted in internalisation of $\beta$-catenin, which was more pronounced for $\mathrm{BIO}$ treatment (Figure $2 \mathrm{~F}$ ).
Migration of paediatric HGG cells in transwell assays is inhibited after treatment with $\mathrm{LiCl}$ and BIO. In order to confirm the anti-migratory effects of $\mathrm{LiCl}$ and $\mathrm{BIO}$ observed in the spheroid model, a 2D transwell assay was performed. Both pHGG cell lines were able to migrate through the transwell membranes, with SF188 demonstrating the most migration over $4 \mathrm{~h}$ (data not shown). In the transwell experiments, treatment with $20 \mathrm{~mm} \mathrm{LiCl}$ and $5 \mu \mathrm{M}$ BIO reduced cell migration in both SF188 and KNS42 confirming the results obtained from the spheroid assays (Figure 3A). Five micromoles of BIO reduced migration in both pHGG cell lines and reached statistical significance for KNS42. Treatment with $20 \mathrm{~mm} \mathrm{LiCl}$ also reduced migration in both cell lines and reached statistical significance for SF188. We conclude that at concentrations of $20 \mathrm{~mm}$ and $5 \mu \mathrm{m}$ respectively, $\mathrm{LiCl}$ and $\mathrm{BIO}$ have significant anti-migratory effects on pHGG cells. Consequently, all subsequent migration assays and IF studies were performed on cells treated with $\mathrm{LiCl}$ or $\mathrm{BIO}$ at these concentrations. Additionally, at the same highest concentrations of $\mathrm{NaCl}$ or DMSO, no adverse effects on cell biology were observed; thus, we carried out all subsequent experiments using growth medium (Dulbecco's Modified Eagles' medium plus 10\% heatinactivated foetal calf serum) as the control.

Next, we assessed the effect of $\mathrm{LiCl}$ and $\mathrm{BIO}$ treatment on cell monolayer viability via a WST-1 assay over a time course of $72 \mathrm{~h}$ (Figure 3B). SF188 was the most sensitive cell line to both treatments. At $24 \mathrm{~h}$, which would most closely correlate to the time scale for the transwell assay, both cell lines demonstrated greater than $80 \%$ viability when treated with $\mathrm{LiCl}$. SF188 was more sensitive to $10 \mu \mathrm{M} \mathrm{BIO}$ at $24 \mathrm{~h}$, with cell viability at $57 \%$; however, KNS42 was resistant to the effects of BIO with greater than $90 \%$ cell viability demonstrated at $24 \mathrm{~h}$. Over $72 \mathrm{~h}$, when cultured as monolayers, both pHGG cell lines exhibited increased sensitivity to $\mathrm{LiCl}$ and $\mathrm{BIO}$ treatment compared with spheroid cultures. This is unsurprising because 3D aggregates of tumour cells, including pHGG cells, have been shown to be generally more drug resistant than 2D cultures (Mehta et al, 2012; Smith et al, 2012). Finally, to demonstrate that $\mathrm{LiCl}$ and $\mathrm{BIO}$ are not directly cytotoxic at concentrations of $20 \mathrm{~mm}$ and $5 \mu \mathrm{M}$ respectively, monolayers of both pHGG cell lines were imaged by time lapse microscopy following treatment with $\mathrm{LiCl}$ and $\mathrm{BIO}$ for $24 \mathrm{~h}$. After $24 \mathrm{~h}$, the drugs were removed, and both pHGG cell lines demonstrated recovery and resumed their normal morphology (results not shown).

$\mathrm{LiCl}$ and BIO inhibit paediatric HGG cell migration on a topographic nanofibre-based migration assay. A nanofibre-based 

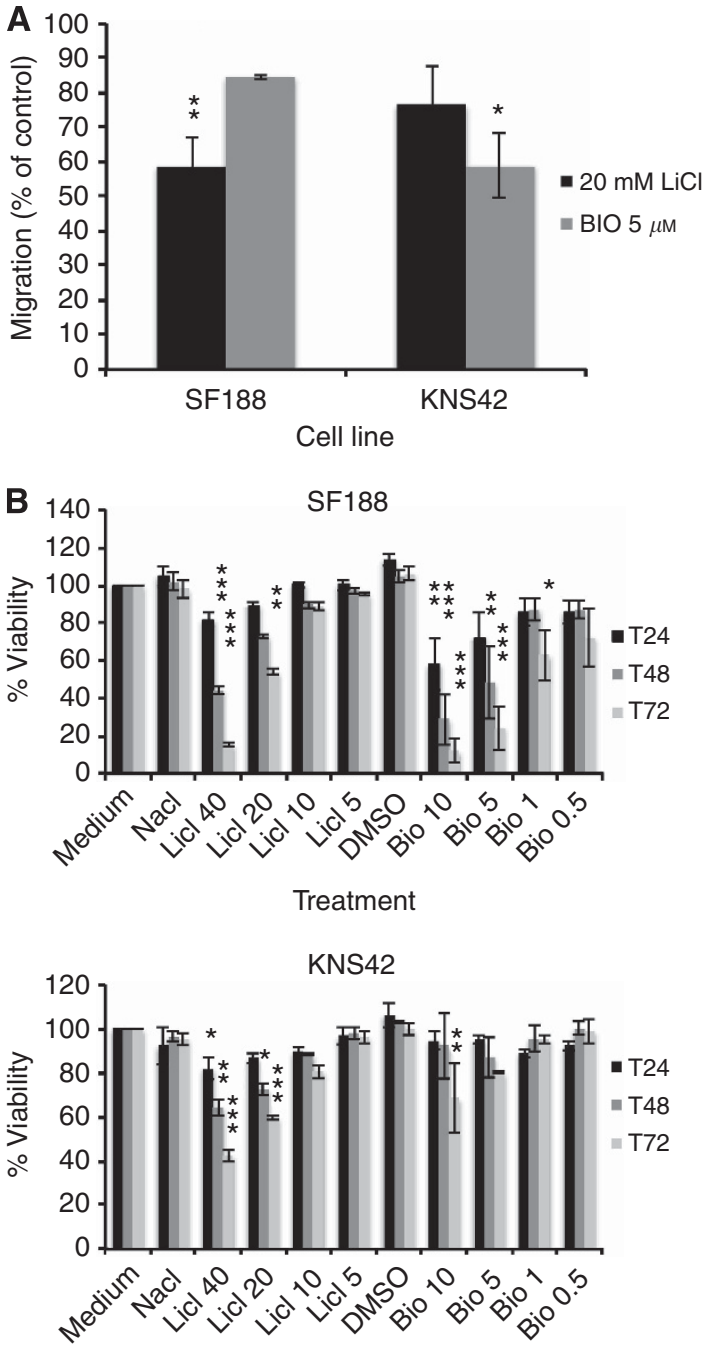

Treatment

Figure 3. $\mathrm{LiCl}$ and $\mathrm{BIO}$ inhibit migration of pHGG cells in a 2D Transwell assay. (A) Cells pre-treated with either control medium, $\mathrm{LiCl}$ and $\mathrm{BIO}$ were assessed for their ability to migrate across a transwell membrane after $4 \mathrm{~h}$ incubation. At $20 \mathrm{~mm} \mathrm{LiCl}$ or $5 \mu \mathrm{M} \mathrm{BIO}$, inhibition of migration was observed in SF188 and KNS42. Results are representative of $n=3$ individual experiments. Error bars are expressed as \pm s.e.m. ${ }^{\star} P<0.05,{ }^{\star \star} P<0.01$ by ANOVA for each cell type and treatment. (B) The effect of $\mathrm{LiCl}$ and $\mathrm{BIO}$ at various concentrations on the paediatric glioma lines (SF188 and KNS42) grown as monolayers was assessed by a WST-1 assay and was expressed as a percentage of controls. Results are representative of $n=3$ individual experiments. Error bars expressed as \pm s.e.m. ${ }^{\star} P<0.05,{ }^{\star \star} P<0.01,{ }^{\star} * \star P<0.001$ by ANOVA for each cell type and treatment.

assay was also used to assess the effect of GSK-3 inhibition on migration of pHGG cell lines on a 3D scaffold. This assay utilises aligned fibres of polycaprolactone to stimulate cell migration via topographic cues (Johnson et al, 2009). When cells are plated on the nanofibre scaffold they adhere, elongate and move along the fibre axis (Figure $4 \mathrm{~A}$ ).

Firstly, we noted that each pHGG cell line was able to migrate in this model system and further confirmation of the anti-migratory effect of the GSK-3 inhibitors $\mathrm{LiCl}$ and $\mathrm{BIO}$ was obtained. As seen in previous assays, SF188 was the most migratory cell line in terms of distance travelled. Perimeter indices (\% increase from the spheroid perimeter to the perimeter marking the migrated cells) were calculated for each cell line. Treatment with $\mathrm{LiCl}$ and $\mathrm{BIO}$ decreased the migration index (Figure 4B) and perimeter index (Figure 4C) for both pHGG cell lines when compared with controls. However, the effect was more marked for KNS42 when compared with SF188.

Live cell imaging of paediatric HGG cell line migration. Given the accumulation of evidence that $\mathrm{LiCl}$ and $\mathrm{BIO}$ are able to reduce the migration of SF188 and KNS42 cells, we went on to hypothesise that we should be able to detect direct changes in cell morphology and motility following treatment. We investigated the effects of $\mathrm{LiCl}$ and $\mathrm{BIO}$ on individual cell morphology by live cell imaging over $24 \mathrm{~h}$. SF188 and KNS42 cells were incubated with $20 \mathrm{~mm} \mathrm{LiCl}$, $5 \mu \mathrm{M}$ BIO or control medium and time lapse imaging was performed for $24 \mathrm{~h}$ (Figure 5A and B). In terms of motility, SF188 cells were more motile and demonstrated greater velocity than KNS42 cells (mean \pm s.e.m. velocity SF188 control $0.295 \pm 0.0018 \mu \mathrm{m} \mathrm{min}^{-1} \mathrm{KNS} 42$ control $0.0599 \pm 0.0048 \mu \mathrm{m} \mathrm{min}^{-1}$ ) (Figure 5C-E). Cells from both lines were able to polarise under normal conditions; however, treatment with $20 \mathrm{~mm} \mathrm{LiCl}$ and $5 \mu \mathrm{M}$ $\mathrm{BIO}$ reduced polarity as demonstrated by a reduction in displacement, but this did not reach statistical significance (displacement SF188 control 176.5 $\pm 74.6, \mathrm{LiCl} 20 \mathrm{~mm} 52.5 \pm 15.3$, BIO $5 \mu \mathrm{mol} 107.2 \pm 61.7$, KNS42 control $28.6 \pm 8.23$, LiCl $20 \mathrm{~mm}$ $14.7 \pm 2.35$, BIO $5 \mu \mathrm{mol} 14.19 \pm 1.9$ ). Twenty millimoles of $\mathrm{LiCl}$ and $5 \mu \mathrm{M}$ BIO reduced the velocity of SF188 cells (control $0.295 \pm 0.0018 \mu \mathrm{m} \mathrm{min}^{-1}, \quad \mathrm{LiCl} 20 \mathrm{mM} \quad 0.153 \pm 0.047 \mu \mathrm{m} \mathrm{min}^{-1}$ $P=0.049, \quad$ BIO $\left.\quad 5 \mu \mathrm{mol} \quad 0.21 \pm 0.041 \mu \mathrm{m} \mathrm{min}^{-1} \quad P=0.247\right)$ whereas treatment only with $5 \mu \mathrm{M}$ BIO reduced the velocity of KNS42 cells (control $0.0599 \pm 0.0048 \mu \mathrm{m} \mathrm{min}^{-1}$, $\mathrm{LiCl}$ $20 \mathrm{~mm} 0.0761 \pm 0.0063 \mu \mathrm{m} \mathrm{min}^{-1} P=0.98$, BIO $5 \mu \mathrm{mol} 0.0501 \pm$ $\left.0.041 \mu \mathrm{m} \mathrm{min}^{-1} P=0.345\right)$. $\mathrm{LiCl}$ and BIO appeared to have a greater effect on the polarity of KNS42 cells than velocity. Apart from the effects on cell motility, both $\mathrm{LiCl}$ and $\mathrm{BIO}$ appeared to affect cell morphology with cells rounding up after treatment and demonstrating loss of polarity and blebbing (Figure 5A and B). Non-apoptotic plasma membrane blebbing has been implicated in cell movement, mode of migration and disruption of actinmembrane interactions (Fackler and Grosse, 2008). Taken together, these observations may explain why the cells incubated with these drugs demonstrated a reduction in migration in the previous experiments.

Immunofluorescence studies reveal differences in cellular morphology in paediatric HGG cell lines and morphological changes after drug treatment. We next utilised IF techniques to investigate the effects of the GSK-3 inhibitors on the microtubule cytoskeleton (polarity), actin cytoskeleton (morphology, motility) and focal adhesions (cell motility, morphology). Both cell lines were characterised by distinctive microtubule network, actin cytoskeleton and focal adhesion staining patterns. Immunofluorescence labelling revealed either elaborate microtubule networks across the whole cell body or a more pronounced cell surface localisation, whereas for actin we observed stress fibres, cortical actin or a diffuse internal localisation ('other') and whole body, cortical or diffuse focal adhesion labelling, which changed in response to treatment. Untreated SF188 cells appeared as large cells with a microtubule network, pronounced actin stress fibres and focal adhesions across the whole cell body (Figure 6A). After treatment with $20 \mathrm{~mm} \mathrm{LiCl}$ and $5 \mu \mathrm{M} \mathrm{BIO}$, we observed that the cells appeared more rounded with pronounced microtubule localisation on the cell surface. Actin stress fibre labelling in the control cells was marked and decreased in the cells treated with $\mathrm{LiCl}$ and $\mathrm{BIO}$ with a more diffuse cortical and internal actin distribution ('other'). (Figure 6C). Focal adhesions across the cell body were diminished after treatment with the inhibitors and, when present, re-distributed to the surface of the cells (Figure 6D). KNS42 cells appeared as medium-sized elongated cells with a microtubule network and had diffuse actin stress fibres (Figure 6B). 



Figure 4. $\mathrm{LiCl}$ and $\mathrm{BIO}$ reduce migration of pHGG cells in an aligned nanofibre 3D migration assay. Tumour cell aggregates formed from hanging

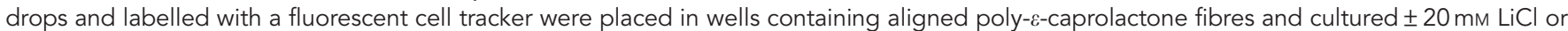
$5 \mu \mathrm{M} \mathrm{BIO}$. Migration was recorded either as phase contrast or fluorescent images for quantification. (A) Spheroids formed from cell line SF188 migrated along the nanofibre as evidenced by phase contrast and fluorescent imaging; the addition of LiCl or $\mathrm{BIO}$ had a marked anti-migratory effect on the cells. Images at $\times 40$ magnification. Scale bar $=400 \mu \mathrm{m}$. (B) Quantification and comparison of the effects of $20 \mathrm{~mm} \mathrm{LiCl}$ and $5 \mu \mathrm{M} \mathrm{BIO}$ on the migration index for both cell lines. Results representative of at least three repeats. Error bars are expressed as \pm s.e.m. ${ }^{\star} P<0.05,{ }^{\star \star} P<0.01$, $\star \star \star P<0.001$ by ANOVA for each cell type and treatment. (C) Quantification and comparison of the effects of $20 \mathrm{~mm} \mathrm{LiCl} \mathrm{and} 5 \mu \mathrm{M} \mathrm{BIO} \mathrm{on} \mathrm{the}$ perimeter index for both cell lines. Error bars are expressed as \pm s.e.m. ${ }^{\star} P<0.05,{ }^{\star \star} P<0.01,{ }^{\star \star \star} P<0.001$ by ANOVA for each cell type and treatment.

Focal adhesions appeared to be associated with actin filaments in untreated cells. After addition of the inhibitors, the cells rounded up and were smaller in comparison to the untreated cells. The microtubule network was still present, however, actin stress fibres appeared to be less pronounced (especially after treatment with $\mathrm{BIO}$ ) (Figure 6C) and focal adhesions co-localised diffusely with the actin filament and when present relocated to the cortex (Figure 6D).

\section{DISCUSSION}

Paediatric high grade glioma and DIPG are devastating tumours associated with poor prognosis and despite attempts at aggressive therapy, tumours inevitably recur owing to their diffuse and invasive nature. As a result, there is a need to identify and develop novel therapeutic approaches that target and block tumour invasion. In this study, we characterised the migratory behaviour of two pHGG cell lines and one patient-derived DIPG cell line in
$2 \mathrm{D}$ and $3 \mathrm{D}$ models and investigated their response to GSK-3 inhibitors. We have shown that different paediatric glioma cell lines are able to grow as tumour spheroids, which are capable of migrating through a collagen matrix. To the best of our knowledge, this is the first time that DIPG migration has been described using a 3D model. Each cell line investigated demonstrated a unique pattern of migration and invasion. We also examined the motility of individual pHGG cells via live cell imaging, which indicated different speeds and morphology in two cell lines. In addition, we have demonstrated for the first time that $\mathrm{LiCl}$ and $\mathrm{BIO}$, which have anti-invasive effects in adult glioma cell lines (Nowicki et al, 2008; Williams et al, 2011), also inhibit the migration of paediatric glioma cells in 2D (transwell) and 3D (spheroid invasion assay and nanofibre plates) assays. We observed a significant inhibition of migration with $5 \mu \mathrm{M} \mathrm{BIO}$ and $20 \mathrm{~mm} \mathrm{LiCl}$ in all of our paediatric glioma cell lines. This was not accompanied by marked loss of viability when SF188 and KNS42 cells were cultured as multicellular spheroids. Our data correlate with previously published results for these inhibitors in adult glioma models (Nowicki et al, 


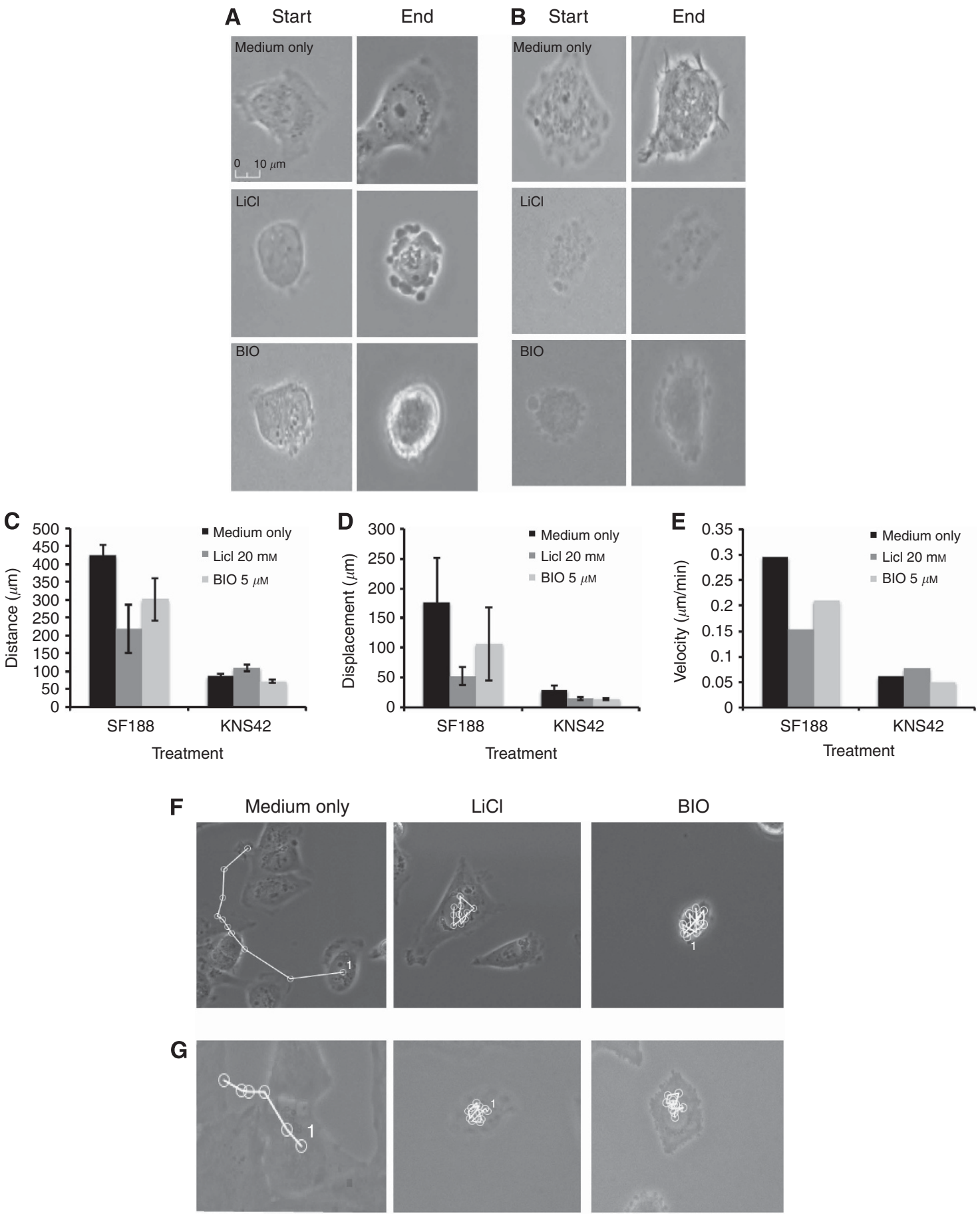

Figure 5. Live cell imaging of pHGG cell lines reveals differences in migration velocity alongside changes in polarity and morphological appearance following treatment with $\mathrm{LiCl}$ and $\mathrm{BIO}$. Paediatric glioma cell lines SF188 and KNS42 were incubated with $20 \mathrm{~mm} \mathrm{LiCl}, 5 \mu \mathrm{M} \mathrm{BIO} \mathrm{or}$ growth medium and time lapse imaging was performed for $24 \mathrm{~h}$ using the Nikon Biostation IM time lapse imaging system. Stills taken at time $0 \mathrm{~h}$ and $24 \mathrm{~h}$ from movies of SF188 (A) and KNS42 (B) treated with either $20 \mathrm{~mm} \mathrm{LiCl,} 5 \mu \mathrm{M} \mathrm{BIO}$ or growth medium demonstrate changes in the morphological appearance of individual cells following treatment. Quantification analysis of distance (C) displacement (D) and velocity (E) from live cell imaging of both cell lines following treatment with $20 \mathrm{~mm} \mathrm{LiCl}$ or $5 \mu \mathrm{m} \mathrm{BIO}$. Error bars are expressed as \pm s.e.m. ${ }^{\star} P<0.05$ by ANOVA for each cell type and treatment. Tracking analysis of SF188 (F) and KNS42 (G) from live cell imaging following treatment with $20 \mathrm{~mm} \mathrm{LiCl}$ or $5 \mu \mathrm{M} \mathrm{BIO}$ demonstrates differences in motility between cell lines and treatments.

2008; Williams et al, 2011). Finally, we have shown that $\mathrm{LiCl}$ and BIO treatment altered the motility of individual pHGG cells, resulting in overall reduced movement, loss of polarity and cell rounding. This observation is novel and demonstrates the specific effects of $\mathrm{LiCl}$ and $\mathrm{BIO}$ on cell morphology and motility. Overall, our study is amongst the very few to have identified existing clinical and preclinical agents, which are capable of disrupting migration and invasion in pHGG and DIPG cell lines.

Cell migration may be achieved by a set of component processes, which are often regulated by the same effectors regardless of the cell type and the mode of migration. Broadly, these processes are based on polarisation, protrusion and adhesion, 

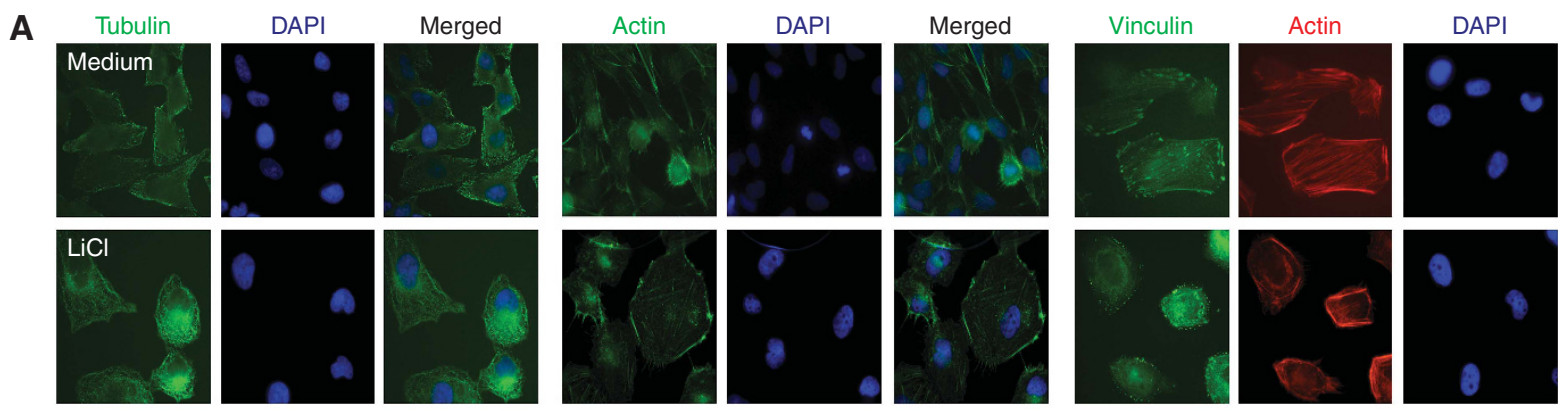

Merged


Figure 6. Immunofluorescence studies reveal cytoskeletal changes in two pHGG cell lines after $\mathrm{LiCl}$ and $\mathrm{BIO}$ treatment. The effects of the two inhibitors $\mathrm{LiCl}$ and $\mathrm{BIO}$ on cell morphology of (A) SF188 and (B) KNS42 were assessed by immunofluorescent labelling. Green labelling-alphatubulin, actin or vinculin; red labelling-actin and blue labelling-DAPI staining. Magnification $\times 63$; changes in the distribution of (C) the actin cytoskeleton (cortical, stress fibres, 'other') and (D) focal adhesions (cortical, diffuse, whole cell body) were observed following scoring of at least 100 cells. 
translocation of the cell body and retraction of the rear. They are also coordinated and integrated by extensive transient signalling networks (Wehrle-Haller and Imhof, 2003). We therefore investigated the effect of $\mathrm{LiCl}$ and $\mathrm{BIO}$ on the interaction between the microtubule cytoskeleton, actin skeleton and focal adhesions on two established pHGG cell lines that grow as monolayers. In both pHGG cell lines examined, we observed that microtubules relocalised to the surface of cells. There was a change in the actin cytoskeleton with re-localisation of the actin fibres to the cell periphery and we also noted a change in focal adhesion point turn over and dynamics with a marked re-distribution of focal adhesion points to the cell surface or apparent loss of focal adhesion foci. The cells appeared to be immobilised in response to the loss of polarity, motility and attachment-detachment dynamics, accounting for their inability to migrate.

One postulated mechanism for the anti-migratory effects of $\mathrm{LiCl}$ and BIO is through GSK-3 inhibition. Glycogen synthase kinase-3 is thought to be involved in the control of cell migration, through its role in regulating cellular structure alongside microtubule and focal adhesion dynamics (Grimes and Jope, 2001; Sun et al, 2009). In adult $\mathrm{HGG}$, the effect of $\mathrm{LiCl}$ and $\mathrm{BIO}$ on migration has previously been investigated and their anti-migratory activity has been attributed to their ability to inhibit GSK-3 and stabilise $\beta$-catenin via the canonical Wnt signalling pathway (Luo, 2009). In adult glioma models, $\mathrm{LiCl}$ has been shown to increase $\beta$-catenin reporter activity and $\beta$-catenin knockdown has been demonstrated to rescue the anti-migratory effects of BIO (Nowicki et al, 2008; Williams et al, 2011). In our study, we examined $\beta$-catenin localisation by IF post treatment of pHGG cells with $\mathrm{LiCl}$ and $\mathrm{BIO}$, and observed a marked internalisation of $\beta$-catenin to the cytoplasm and nucleus following treatment. This observation has also been noted in adult glioma following $\mathrm{LiCl}$ and $\mathrm{BIO}$ treatment (Williams, 2011) and provides evidence to support the effectiveness of $\mathrm{LiCl}$ and $\mathrm{BIO}$ as an inhibitor of GSK-3. Furthermore, western blot analysis of our pHGG lines confirmed that $\mathrm{LiCl}$ increased Ser9 phosphorylated GSK- $3 \beta$ (inactivated form) and BIO decreased the activating tyrosine of GSK- $3 \alpha \beta$.

Both $\mathrm{LiCl}$ and $\mathrm{BIO}$ have potential for clinical application. $\mathrm{LiCl}$ has been used to treat psychiatric conditions such as bipolar disorder for many decades (Mitchell, 1999) and BIO is part of the family of indirubins, which have been identified as the active ingredient of the Chinese medicine Danggui Longhui Wan, which has anti-leukemic activity (Hoessel et al, 1999). Other GSK-3 inhibitors have been developed and tested in a variety of preclinical studies (Meijer et al, 2004; Eldar-Finkelman and Martinez, 2011), however, current hurdles for the use of such drugs in clinical practice include optimisation of drug delivery and avoidance of toxic systemic accumulation of the drug. In particular, $\mathrm{LiCl}$ has a narrow therapeutic window, inducing nephrotoxicity at concentrations greater than $1.5 \mathrm{~mm}$ in vivo (Nowicki et al, 2008). Although our data detect significant anti-migratory effects on paediatric glioma at concentrations as low as $5 \mathrm{~mm}$, alternative methods of drug delivery such as convection-enhanced delivery or local delivery with polymers may be required to attain localised high concentrations of the drug in human brain tumours (Nowicki et al, 2008; Siegal, 2013) and this requires further evaluation in vivo with preclinical models. Alternatively, the development of specific novel GSK-3 inhibitors capable of crossing the blood-brain barrier at concentrations associated with clinically acceptable side effect profiles will help overcome this problem. Finally, owing to the lack of published mouse models of paediatric glioma invasion, we have not been able to address the anti-migratory effects of GSK-3 inhibitors in vivo. Studies of adult glioma have shown that GSK-3 inhibitors of the indirubin family are able to reduce invasion of glioma cells in vivo (Williams et al, 2011) and development of a paediatric orthotopic xenograft model of migration to test novel GSK-3 inhibitors forms a major part of our ongoing studies in this area.
In summary, we have characterised the migratory behaviour of paediatric glioma cell lines in 2D and 3D models and conclude that GSK-3 inhibitors, such as $\mathrm{LiCl}$ and $\mathrm{BIO}$, may be novel candidates for migration inhibition in $\mathrm{pHGG}$ and DIPG and as such warrant further investigation as therapeutics for this challenging group of tumours.

\section{ACKNOWLEDGEMENTS}

We would like to thank our funders Yorkshire Cancer Research, the PPR Foundation, Candlelighters Children Cancer Charity and Brain Tumour Research and Support across Yorkshire who have helped support this work.

\section{CONFLICT OF INTEREST}

The authors declare no conflict of interest.

\section{REFERENCES}

Agudelo-Garcia PA, De Jesus JK, Williams SP, Nowicki MO, Chiocca EA, Liyanarachchi S, Li PK, Lannutti JJ, Johnson JK, Lawler SE, Viapiano MS (2011) Glioma cell migration on three-dimensional nanofiber scaffolds is regulated by substrate topography and abolished by inhibition of STAT3 signaling. Neoplasia 13: 831-840.

Broniscer A, Gajjar A (2004) Supratentorial high-grade astrocytoma and diffuse brainstem glioma: two challenges for the pediatric oncologist. Oncologist 9: 197-206.

Del Duca D, Werbowetski T, Del Maestro RF (2004) Spheroid preparation from hanging drops: characterization of a model of brain tumor invasion. J Neurooncol 67: 295-303.

Demuth T, Berens ME (2004) Molecular mechanisms of glioma cell migration and invasion. J Neurooncol 70: 217-228.

Eldar-Finkelman H, Martinez A (2011) GSK-3 inhibitors: preclinical and clinical focus on CNS. Front Mol Neurosci 4: 32.

Fackler OT, Grosse R (2008) Cell motility through plasma membrane blebbing. J Cell Biol 181: 879-884.

Grimes CA, Jope RS (2001) The multifaceted roles of glycogen synthase kinase 3beta in cellular signaling. Prog Neurobiol 65: 391-426.

Hoessel R, Leclerc S, Endicott JA, Nobel ME, Lawrie A, Tunnah P, Leost M, Damiens E, Marie D, Marko D, Niederberger E, Tang W, Eisenbrand G, Meijer L (1999) Indirubin, the active constituent of a Chinese antileukaemia medicine, inhibits cyclin-dependent kinases. Nat Cell Biol 1: $60-67$.

Johnson J, Nowicki MO, Lee CH, Chiocca EA, Viapiano MS, Lawler SE, Lannutti JJ (2009) Quantitative analysis of complex glioma cell migration on electrospun polycaprolactone using time-lapse microscopy. Tissue Eng Part C Methods 15: 531-540.

Jones C, Perryman L, Hargrave D (2012) Paediatric and adult malignant glioma: close relatives or distant cousins? Nat Rev Clin Oncol 9: 400-413.

Louis DN (2006) Molecular pathology of malignant gliomas. Annu Rev Pathol 1: $97-117$.

Luo J (2009) Glycogen synthase kinase 3beta (GSK3beta) in tumorigenesis and cancer chemotherapy. Cancer Lett 273: 194-200.

Ma XH, Piao S, Wang D, McAfee QW, Nathanson KL, Lum JJ, Li LZ, Amaravadi RK (2011) Measurements of tumor cell autophagy predict invasiveness, resistance to chemotherapy, and survival in melanoma. Clin Cancer Res 17: 3478-3489.

Mehta G, Hsiao AY, Ingram M, Luker GD, Takayama S (2012) Opportunities and challenges for use of tumor spheroids as models to test drug delivery and efficacy. J Control Release 164: 192-204.

Meijer L, Flajolet M, Greengard P (2004) Pharmacological inhibitors of glycogen synthase kinase 3. Trends Pharmacol Sci 25: 471-480.

Mitchell PB (1999) On the 50th anniversary of John Cade's discovery of the anti-manic effect of lithium. Aust N Z J Psychiatry 33: 623-628.

Nowicki MO, Dmitrieva N, Stein AM, Cutter JL, Godlewski J, Saeki Y, Nita M, Berens ME, Sander LM, Newton HB, Chiocca EA, Lawler S (2008) Lithium 
inhibits invasion of glioma cells; possible involvement of glycogen synthase kinase-3. Neuro Oncol 10: 690-699.

Siegal T (2013) Which drug or drug delivery system can change clinical practice for brain tumor therapy? Neuro Oncol 15: 656-669.

Smith SJ, Wilson M, Ward JH, Rahman CV, Peet AC, Macarthur DC, Rose FR, Grundy RG, Rahman R (2012) Recapitulation of tumor heterogeneity and molecular signatures in a $3 \mathrm{D}$ brain cancer model with decreased sensitivity to histone deacetylase inhibition. PLoS One 7: e52335.

Sun T, Rodriguez M, Kim L (2009) Glycogen synthase kinase 3 in the world of cell migration. Dev Growth Differ 51: 735-742.

Van Beusechem VW, Mastenbroek DC, van den Doel PB, Lamfers ML, Grill J, Würdinger T, Haisma HJ, Pinedo HM, Gerritsen WR (2003) Conditionally replicative adenovirus expressing a targeting adapter molecule exhibits enhanced oncolytic potency on CAR-deficient tumors. Gene Ther 10: 1982-1991.

Vinci M, Gowan S, Boxall F, Patterson L, Zimmermann M, Court W, Lomas C, Mendiola M, Hardisson D, Eccles SA (2012) Advances in establishment and analysis of three-dimensional tumor spheroid-based functional assays for target validation and drug evaluation. BMC Biol 10: 29 .
Wakefield JG, Stephens DJ, Tavaré JM (2003) A role for glycogen synthase kinase-3 in mitotic spindle dynamics and chromosome alignment. J Cell Sci 116: 637-646.

Warren KE (2012) Diffuse intrinsic pontine glioma: poised for progress. Front Oncol 2: 205.

Wehrle-Haller B, Imhof BA (2003) Actin, microtubules and focal adhesion dynamics during cell migration. Int J Biochem Cell Biol 35: 39-50.

Williams SP (2011) The role of glycogen synthase kinase in glioblastoma multiforme migration and invasion. $\mathrm{PhD}$ thesis, The Ohio State University: Columbus, OH, USA.

Williams SP, Nowicki MO, Liu F, Press R, Godlewski J, Abdel-Rasoul M, Kaur B, Fernandez SA, Chiocca EA, Lawler SE (2011) Indirubins decrease glioma invasion by blocking migratory phenotypes in both the tumor and stromal endothelial cell compartments. Cancer Res 71: 5374-5380.

This work is published under the standard license to publish agreement. After 12 months the work will become freely available and the license terms will switch to a Creative Commons AttributionNonCommercial-Share Alike 4.0 Unported License.

Supplementary Information accompanies this paper on British Journal of Cancer website (http://www.nature.com/bjc) 